\title{
Association of Epstein-Barr virus serological reactivation with transitioning to systemic lupus erythematosus in at-risk individuals
}

\author{
Neelakshi R Jog, ${ }_{1}^{1}$ Kendra A Young, ${ }^{2}$ Melissa E Munroe, ${ }^{1}$ Michael T Harmon, ${ }^{1}$ \\ Joel M Guthridge, ${ }^{1}$ Jennifer A Kelly, ${ }^{1}$ Diane L Kamen, ${ }^{3}$ Gary S Gilkeson, ${ }^{3}$ \\ Michael H Weisman, ${ }^{4}$ David R Karp, ${ }^{5}$ Patrick M Gaffney, ${ }^{1}$ John B Harley, ${ }^{6,7,8}$ \\ Daniel J Wallace, ${ }^{4}$ Jill M Norris, ${ }^{2}$ Judith A James ${ }^{\oplus 1,9}$
}

Handling editor Josef $S$ Smolen

- Additional material is published online only. To view please visit the journal online (http://dx.doi.org/10. 1136annrheumdis-2019215361)

For numbered affiliations see end of article.

\section{Correspondence to}

Dr Judith A James, Arthritis \& Clinical Immunology, Oklahoma Medical Research Foundation, Oklahoma City, OK 73104, USA judith-james@omrf.org

Received 10 March 2019 Revised 6 May 2019 Accepted 17 May 2019

Published Online First 19 June 2019

\section{ABSTRACT}

Objective Systemic lupus erythematosus (SLE) is a systemic autoimmune disease with unknown aetiology. Epstein-Barr virus (EBV) is an environmental factor associated with SLE. EBV maintains latency in B cells with frequent reactivation measured by antibodies against viral capsid antigen (VCA) and early antigen (EA). In this study, we determined whether EBV reactivation and single nucleotide polymorphisms (SNPS) in EBVassociated host genes are associated with SLE transition. Methods SLE patient relatives $(n=436)$ who did not have SLE at baseline were recontacted after $6.3( \pm 3.9)$ years and evaluated for interim transitioning to SLE ( $\geq 4$ cumulative American College of Rheumatology criteria); $56(13 \%)$ transitioned to SLE prior to the follow-up visit. At both visits, detailed demographic, environmental, clinical information and blood samples were obtained. Antibodies against viral antigens were measured by ELISA. SNPs in IL 10, CR2, TNFAIP3 and CD40 genes were typed by ImmunoChip. Generalised estimating equations were used to test associations between viral antibody levels and transitioning to SLE.

Results Mean baseline VCA IgG $(4.879 \pm 1.797$ vs $3.866 \pm 1.795, p=0.0003)$ and $E A \lg (1.192 \pm 1.113$ vs $0.7774 \pm 0.8484, p=0.0236$ ) levels were higher in transitioned compared with autoantibody negative non-transitioned relatives. Increased VCA IgG and EA $\lg$ G were associated with transitioning to SLE (OR 1.28 $95 \% \mathrm{Cl} 1.07$ to $1.53, \mathrm{p}=0.007$, OR 1.43 95\% Cl 1.06 to $1.93, p=0.02$, respectively). Significant interactions were observed between CD40 variant rs48100485 and VCA IgG levels and IL 10 variant rs3024493 and VCA IgA levels in transitioning to SLE.

Conclusion Heightened serologic reactivation of EBV increases the probability of transitioning to SLE in unaffected SLE relatives.

\section{INTRODUCTION}

Systemic lupus erythematosus (SLE) is a systemic autoimmune disease that involves multiple organ systems and causes significant morbidity. The aetiology of SLE is not completely understood. Genetic risk factors have been associated with SLE. ${ }^{2}$ However, monozygotic twin concordance rates of $24 \%-69 \% \%^{34}$ suggest a role for environmental factors in SLE pathogenesis.

\section{Key messages}

What is already known about this subject?

- Significant associations between Epstein-Barr virus (EBV) serological markers and systemic lupus erythematosus (SLE) have been shown.

- Patients with SLE have higher frequencies of EBV-infected cells, higher viral loads and higher early antigen IgG levels.

What does this study add?

- This is a prospective study to understand the contribution of EBV reactivation prior to SLE disease onset in at risk individuals.

- The study shows that previously unaffected relatives of patients with SLE who subsequently develop classified SLE have higher serological reactivation of EBV compared with those who do not transition to SLE.

- The study also shows that variants in genes that are associated with SLE and implicated in EBV infection, associate with transitioning to SLE.

How might this impact on clinical practice or future developments?

- Serological measures of EBV reactivation may provide additional biomarkers to identify individuals at higher risk of transitioning to clinical SLE.

Epstein-Barr virus (EBV) is a gamma-herpes virus that ubiquitously infects the majority of the world's population. EBV maintains latency in B cells and occasionally reactivates the lytic cycle. EBV infection elicits an $\operatorname{IgG}$ response to viral capsid antigen (VCA), followed by an IgG response to early antigen (EA). EA IgG levels are detectable as early as a few months and up to 2 years following infection. VCA IgG responses persist for life, although levels are lower during latency compared with lytic infection. ${ }^{5}$ Reactivation of EBV is proposed to increase VCA IgG levels and also leads to detectable EA IgG levels. ${ }^{5}$ Serology can therefore be used to identify individuals with current infection or recent viral reactivation.

Significant associations between SLE and serological markers of EBV infection, VCA IgG and EA IgG 
have been shown. ${ }^{6-8}$ Patients with SLE have higher frequencies of EBV-infected cells, higher viral loads in blood mononuclear cells and higher levels of EA IgG, ${ }^{9-12}$ suggesting more frequent EBV reactivation. However, whether this recurring EBV reactivation is a consequence of immune dysregulation and/or immune suppression in patients with SLE is not clear. To our knowledge, no prospective studies have been performed to understand the cause/effect relationship between viral reactivation and SLE classification.

The ligation of human complement receptor 2 (CR2) with gp350/220 glycoprotein of EBV leads to endocytosis and subsequent EBV infection of B cells. ${ }^{13} 14$ EBV encodes homologues of human proteins to aid in infection and/or maintain latency. An EBV homologue of interleukin 10 (IL-10) promotes B cell survival and induces pro-inflammatory phenotype in monocytes. ${ }^{15}{ }^{16}$ Latent membrane protein 1, a viral mimic of CD40, induces extrafollicular $\mathrm{B}$ cell differentiation. ${ }^{17} \mathrm{EBV}$ infection induced CD40L/CD40 signalling in host cells may support viral persistence. ${ }^{18}$ Interestingly, candidate gene studies in patients with SLE relative to healthy controls have revealed that single nucleotide polymorphisms (SNPs) in host IL10 (rs3024505, rs3024495, rs3024493 and 3122605) and CD40 (rs1569723, rs1883832 and rs4810485) and a three SNP CR2 haplotype (rs3813946, rs1048971 and rs17615) are associated with lupus susceptibility. ${ }^{19-23}$ Relationships between EBV infection/reactivation and genetic variants in host genes implicated in these viral-associated pathways in SLE are unknown.

In this study, we examined whether EBV serological reactivation precedes SLE classification and whether the associations between serological measures of EBV reactivation and transitioning to SLE were modified by variants in host EBV-related genes. Interactions between EBV reactivation measures and SNPs in TNFAIP3, which is associated with SLE but not involved in EBV infection in SLE transitioning were also determined.

\section{METHODS}

\section{Study design and patients}

Study participants were previously enrolled in the Lupus Family Registry and Repository and Systemic Lupus Erythematosus in Gullah Health studies ${ }^{24} 25$ and provided consent for recontact. This study analysed baseline samples of individuals $(n=436)$ who had a relative with SLE and who did not meet $\geq 4$ American College of Rheumatology (ACR) criteria for SLE at the time of their baseline visit. A follow-up study assessed ACR criteria to determine interim transition to classified SLE in these previously unaffected relatives, hereafter referred to as non-transitioned ( $<4$ ACR criteria at follow-up) or transitioned ( $\geq 4$ ACR criteria at follow-up) relatives. Unrelated unaffected controls, hereafter referred to as controls $(n=122)$, were recruited at local health fairs in Oklahoma and were age and sex matched to SLE relatives. All study participants provided informed consent prior to enrolment. All protocols were approved by the Oklahoma Medical Research Foundation (OMRF) or Medical University of South Carolina Institutional Review Boards to be compliant with the Helsinki Declaration.

\section{Patient and public involvement}

Research was done without patient involvement in the study design, data analysis/interpretation or writing/editing of this manuscript.

\section{Autoantibody and antiviral response testing}

Autoantibody testing was performed by the College of American Pathologists (CAP)-certified/Clinical Laboratory Improvement Amendments (CLIA)-approved OMRF Clinical Immunology Laboratory as described previously. ${ }^{26} 27$ Antinuclear antibodies (ANA) and antidouble-stranded DNA (dsDNA) antibodies were determined by indirect immunofluorescence using Hep2 cells or Crithidia luciliae, respectively (INOVA Diagnostics, San Diego, California, USA). Positivity was defined as ANA detection at a titre of $\geq 1: 120$ and anti-dsDNA detection at $\geq 1: 30$. Antibodies against extractable nuclear antigens (Ro, La, Sm, nRNP and ribosomal P) were detected by immunodiffusion. Anticardiolipin antibodies were measured by ELISA. Positivity was defined as $>20$ IgG or IgM units.

Antibodies against EBV VCA (IgG), EBV EA (IgG) (Zeus Scientific (Alere-Wampole/Abbott), Branchburg, New Jersey) and EBV VCA (IgA) (Calbiotech, El Cajon, California, USA) were measured using commercial ELISAs according to manufacturer's instructions in samples collected at the baseline visit for SLE relatives and controls. Antibodies against unrelated herpes viruses, Cytomegalovirus (CMV, IgG) and herpes simplex virus-1 (HSV1, IgG), and varicella zoster (VZV, IgG) (Zeus Scientific (Alere-Wampole/Abbott), Branchburg, New Jersey, USA) were also measured. Standard calibrators were used in each assay to calculate index values/optical density (OD) ratios, which serve as a semiquantitative measure of antibody levels. All assays met predetermined quality control measures based on positive, negative and blank controls. Positivity was defined as OD ratio $\geq 1.1$.

\section{Genotyping study participants}

Five SNPs in IL10, three in CD40, one in CR2 and five in TNFAIP3, previously shown to be associated with autoimmune diseases, were genotyped using the ImmunoChip and were read on the Illumina iScan in the OMRF Clinical Genomics Centre as described previously. ${ }^{28}$ Each SNP was tested for consistency with Hardy-Weinberg proportions using a one-degree of freedom $\chi^{2}$ goodness-of-fit test with a $\mathrm{p}$ value of 0.05 considered as evidence of a departure from Hardy-Weinberg equilibrium. IL10 variants, rs3024505, rs3024495, rs3024493 and rs3122605 were in strong linkage disequilibrium $\left(\mathrm{r}^{2} \geq 0.80\right)$. Similarly, CD40 SNPs, rs1569723, rs1883832 and rs4810485 were in strong linkage disequilibrium $\left(\mathrm{r}^{2}>0.80\right)$. IL10 SNP rs3024493 and CD40 SNP rs4810485 were therefore used as surrogates for other SNPs in IL10 and CD40 genes, respectively.

\section{Statistical analysis}

Viral antibody levels at baseline between transitioned relatives $(n=56)$, non-transitioned relatives $(n=380)$ and control $(n=122)$ groups were compared using the Mann-Whitney U test (GraphPad Prism V.7.02). The percent of individuals positive for antiviral responses in the different groups were compared by Fisher's exact test for categorical variables (GraphPad Prism V.7.02). Generalised estimating equations (GEE), adjusting for correlation within families, were used to test associations between the viral antibody levels and the categorical outcome of transitioning to SLE. ORs are reported for one unit change in antibody level (OD ratio). Associations with SNPs were examined in an additive model treating the number of minor alleles as a continuous variable with the OR representing an increase (or decrease) in risk for each minor allele. OR and 95\% CIs were determined for all models. GEE analyses were performed in SAS V9.4. All multivariable models were adjusted for age, sex and race. 
Table 1 Study participant demographics*

\begin{tabular}{|c|c|c|c|c|}
\hline Variable & Transitioned relatives $n=56$ & $\begin{array}{l}\text { Non-transitioned relatives } \\
\mathrm{n}=380\end{array}$ & Unaffected controls $n=122$ & $P$ value \\
\hline Age at baseline: mean (median) $\pm S D$ & $47.4(48.5) \pm 12.1$ & $47.2(48.0) \pm 15.8 \dagger$ & $51.1(51.0) \pm 12.7$ & 0.01 \\
\hline $\begin{array}{l}\text { Time between recontact (years): mean } \\
\text { (median) } \pm \text { SD }\end{array}$ & $5.9(5.1) \pm 3.5$ & $6.3(5.4) \pm 3.9 \dagger$ & NA & 0.50 \\
\hline Sex: female $(n, \%) \ddagger$ & $49(87.5)$ & $316(83.2)$ & $106(86.9)$ & 0.49 \\
\hline \multicolumn{5}{|l|}{ Race $(n, \%)$} \\
\hline $\begin{array}{l}\text { European American } \\
\text { African American } \\
\text { Native American } \\
\text { Asian/Pacific Islander } \\
\text { Hispanic }\end{array}$ & $\begin{array}{l}43(76.8) \\
9(16.1) \\
4(7.1) \\
0(0.0) \\
0(0.0)\end{array}$ & $\begin{array}{l}279(73.4) \\
55(14.5) \\
18(4.7) \\
17(4.5) \\
11(2.9)\end{array}$ & $\begin{array}{l}89(73.0) \\
15(12.3) \\
9(7.4) \\
5(4.1) \\
4(3.2)\end{array}$ & 0.004 \\
\hline ANA positive baseline $(n, \%) \S$ & $43(76.8)$ & $183(48.2)$ & $15(12.3)$ & $<0.0001$ \\
\hline \multicolumn{5}{|l|}{ Number of baseline ACR Criteria } \\
\hline $\begin{array}{l}0-1 \\
2 \\
3\end{array}$ & $\begin{array}{l}7(12.5) \\
23(41.1) \\
26(46.4)\end{array}$ & $\begin{array}{l}291(76.5) \\
74(19.5) \\
15(4.0)\end{array}$ & ND & $<0.0001$ \\
\hline
\end{tabular}

${ }^{*} \mathrm{P}$ value for trend. Statistically significant values $(p<0.05)$ are in bold.

†Not significant between transitioned and non-transitioned relatives by Mann-Whitney $U$ test. $¥$ Not significant between transitioned and non-transitioned relatives by Fisher's exact test. $\S \mathrm{P}<0.0001$ between transitioned and non-transitioned relatives by Fisher's exact test.

ACR, American College of Rheumatology; ANA, antinuclear antibodies; NA, not applicable; ND, not determined.

\section{RESULTS}

\section{Study cohort}

Nearly $75 \%$ of study participants were of European American descent and more than $85 \%$ were female. No significant differences were observed in average age between transitioned and non-transitioned relatives. Transitioned relatives had a greater number of confirmed ACR criteria at study enrolment, and higher percentages were ANA positive at baseline compared with non-transitioned relatives $(\mathrm{p}<0.0001$, table 1$)$.

\section{Relatives who transitioned to SLE had higher baseline levels of anti-VCA IgG compared with relatives who did not transition to SLE}

To understand the contribution of EBV reactivation to SLE classification, we compared the anti-EBV antibody levels at baseline visit in transitioned and non-transitioned relatives. Transitioned relatives had higher baseline VCA IgG levels compared with non-transitioned relatives $(4.88 \pm 1.8$ vs $4.18 \pm 1.81, \mathrm{p}=0.0086$, figure $1 \mathrm{~A})$ and controls $(4.88 \pm 1.8$ vs $4.25 \pm 1.5, p=0.02)$. A higher percentage of controls were positive for VCA IgG compared with non-transitioned relatives (figure 1B). However, VCA IgG seropositivity at baseline was not different between transitioned and non-transitioned relatives (figure 1B), suggesting that both groups of SLE relatives had similar prior exposure to EBV. The total IgG concentrations were not different between transitioned and non-transitioned relatives, suggesting that the increase in VCA IgG levels is not due to hyperglobulinaemia in the transitioned group (see online supplementary figure $\mathrm{S} 1$ ).

At the baseline visit, the transitioned relatives had higher EA IgG levels compared with controls $(1.19 \pm 1.11$ vs $0.62 \pm 0.64$, $\mathrm{p}<0.0001$ ) (figure 1C). Baseline EA IgG levels were not different between transitioned and non-transitioned relatives. A higher percentage of transitioned relatives were positive for EA IgG compared with controls $(37.5 \%$ vs $13.11 \%$, $\mathrm{p}<0.0005)$ but not compared with non-transitioned relatives (figure 1D). IgA responses against VCA were not different between the groups (figure $1 \mathrm{E}, \mathrm{F}$ ).

\section{Relatives who transitioned had higher levels of VCA IgG and EA IgG compared with ANA negative non-transitioned relatives}

A higher number of transitioned relatives were ANA positive at baseline compared with non-transitioned relatives and controls (table 1). Both VCA IgG $(r=0.15, p=0.002)$ and $E A \operatorname{IgG}$ levels $(r=0.22, p<0.0001)$ correlated with increasing numbers of autoantibody specificities (see online supplementary table 1). To determine whether EBV serological reactivation contributes to ANA positivity, we divided the non-transitioned relatives into ANA positive (ANA+) and ANA negative (ANA-) groups at baseline. Transitioned relatives had significantly higher levels of VCA IgG compared with ANA- non-transitioned relatives $(4.879 \pm 1.797$ vs $3.866 \pm 1.795, p=0.0003$ figure $2 \mathrm{~A})$. Lower percentages of non-transitioned relatives were positive for VCA IgG at baseline compared with controls, irrespective of autoantibody positivity (figure 2B).

Transitioned relatives had higher EA IgG at baseline compared with ANA- non-transitioned relatives $(1.192 \pm 1.113$ vs $0.7774 \pm 0.8484, p=0.0236$, figure $2 \mathrm{C}$ ). Higher percentages of transitioned relatives were positive for EA IgG compared with ANA- but not ANA+ non-transitioned relatives (figure 2D). VCA IgG and EA IgG levels were higher in the ANA+ non-transitioned compared with ANA- non-transitioned relatives (figure 2A,C); however, the levels of total $\operatorname{IgG}$ between these two groups were similar (see online supplementary figure $\mathrm{S1}$ ). These data suggest that increased EBV reactivation, as measured by EA IgG positivity and increased VCA IgG and $\mathrm{EA} \operatorname{IgG}$, may contribute to generation of autoantibodies, eventually contributing to autoimmunity.

Controls in this study had higher seropositivity and antibody levels against CMV and HSV-1 compared with transitioned and non-transitioned relatives (figure 2E-H). However, the levels of 

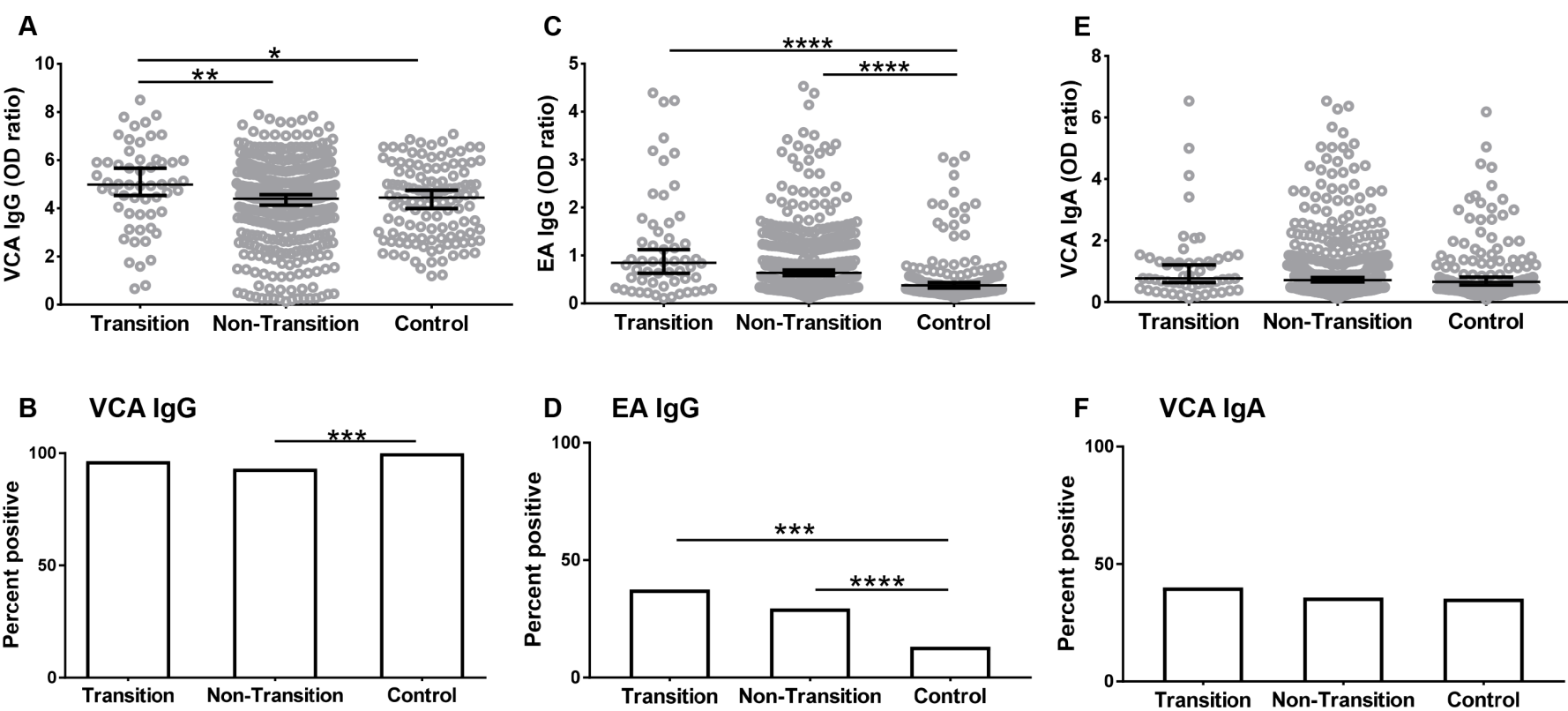

Figure 1 Relatives who subsequently transitioned to SLE had higher baseline levels of anti-EBV IgG compared with healthy controls. IgG antibody levels against EBV VCA (A), EBV EA (C) and IgA antibodies against EBV VCA (E) were measured by ELISA at the baseline visit in relatives who transitioned to SLE (transition), relatives who did not transition to SLE (non-transition) and unaffected unrelated controls (control). Data are represented as median $\pm 95 \% \mathrm{Cl} .{ }^{*} \mathrm{p}<0.05,{ }^{* *} \mathrm{p}<0.01,{ }^{* * * *} \mathrm{p}<0.0001$ by Mann Whitney. Seropositivity for VCA IgG (B), EA IgG (D) and VCA IgA (F) was determined as described in methods. ${ }^{* * *} p<0.001,{ }^{* * *} p<0.0001$ by Fisher's exact test. EA, early antigen; EBV, Epstein-Barr virus; SLE, systemic lupus erythematosus; VCA, viral capsid antigen.

CMV IgG and HSV-1 IgG were not different between transitioned and non-transitioned relatives, irrespective of ANA positivity. No differences in VZV seropositivity or levels of VZV IgG were found between transitioned and non-transitioned relatives nor with healthy controls (see online supplemental figure S2). These data show that the differences with VCA IgG and EA IgG seen between the transitioned and non-transitioned relatives are specific for humoral responses to EBV and not a generalised dysregulation of immune response to herpes viruses in SLE relatives.

\section{Increased levels of VCA IgG and EA IgG associate with transitioning to SLE}

Our data show increased levels of VCA IgG and EA IgG at baseline prior to transitioning to classified disease in relatives of patients with SLE. To determine whether these increased levels associate with transitioning to SLE after adjusting for age, sex and race, we used GEE, accounting for correlation among families. Increasing levels of VCA IgG were associated with transitioning to SLE (table 2).
A
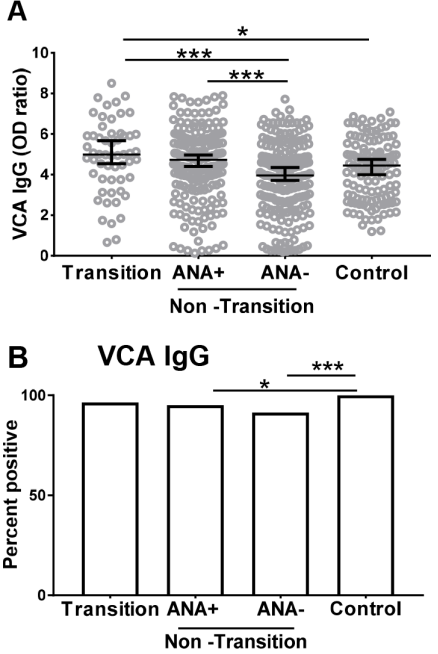

C
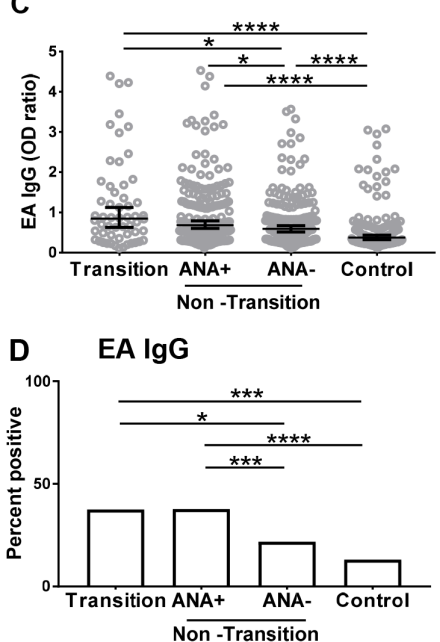

E

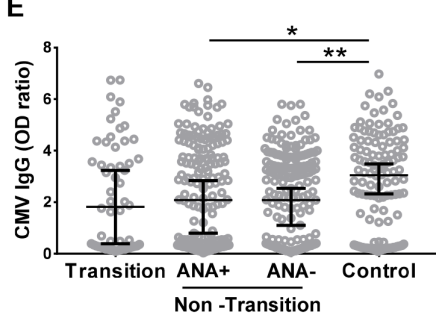

F CMV IgG

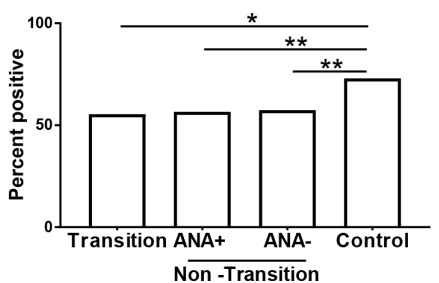

G

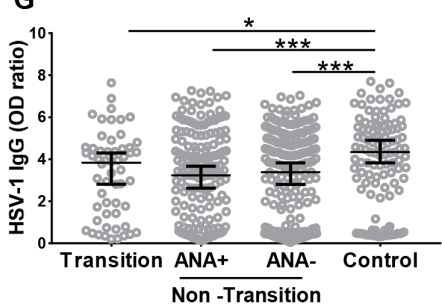

H HSV-1 IgG

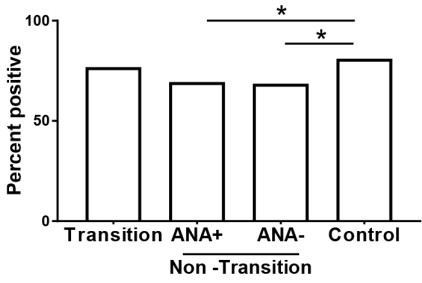

Figure 2 Relatives who transitioned to classified SLE had higher levels of VCA IgG and EA IgG compared with autoantibody negative nontransitioned relatives at baseline. Relatives who did not transition (non-transition) were divided based on their ANA positivity status (as described in methods). Antibody levels $(A, C, E, G)$ and seropositivity $(B, D, F, H)$ for VCA IgG $(A, B), E A \lg G(C, D), C M V \lg G(E, F)$, and HSV-1 IgG $(G, H)$ at the baseline visit were determined by ELISA. Data are represented as median $\pm 95 \% \mathrm{Cl}$. ${ }^{*} \mathrm{p}<0.05,{ }^{* *} \mathrm{p}<0.01,{ }^{* * *} \mathrm{p}<0.001,{ }^{* * * *} \mathrm{p}<0.0001$ by Mann-Whitney $(A, C, E, G)$ or Fisher's exact test $(B, D, F, H)$. ANA, antinuclear antibodies; CMV, cytomegalovirus; EA, early antigen; EBV, Epstein-Barr virus; HSV, herpes simplex virus; SLE, systemic lupus erythematosus; VCA, viral capsid antigen. 
Table 2 Increasing VCA IgG and EA IgG levels are associated with transitioning to SLE

\begin{tabular}{|c|c|c|}
\hline Serological measures & $\begin{array}{l}\text { Transitioned }(n=56) \\
\text { versus non-transitioned } \\
(n=380) \text { relatives } \\
\text { OR }(95 \% C l) \text {, p value }\end{array}$ & $\begin{array}{l}\text { Transitioned relatives } \\
(\mathrm{n}=56) \text { versus unrelated } \\
\text { unaffected controls } \\
(\mathrm{n}=122) \\
\text { OR }(95 \% \mathrm{Cl}) \text {, p value }\end{array}$ \\
\hline VCA IgG (OD ratio) & $\begin{array}{l}1.28 \text { (1.07 to } 1.53), \\
0.007\end{array}$ & 1.30 (1.02 to 1.65$), 0.03$ \\
\hline VCA IgA (OD ratio) & 0.99 (0.78 to 1.27$), 0.94$ & 1.01 (0.73 to 1.42$), 0.91$ \\
\hline EA IgG (OD ratio) & 1.43 (1.06 to 1.93$), 0.02$ & $\begin{array}{l}2.11(1.38 \text { to } 3.23), \\
0.0005\end{array}$ \\
\hline CMV IgG (OD ratio) & 1.03 (0.87 to 1.22$), 0.72$ & 0.89 (0.73 to 1.07$), 0.21$ \\
\hline HSV-1 lgG (OD ratio) & $1.06(0.92$ to 1.22$), 0.44$ & $0.94(0.82$ to 1.09$), 0.43$ \\
\hline
\end{tabular}

Statistically significant values $(p<0.05)$ are in bold

${ }^{*} \mathrm{OR}$ presented for one unit increase in $\mathrm{OD}$ ratio.

CMV, cytomegalovirus; EA, early antigen; HSV, herpes simplex virus; OD, optical

density; SLE, systemic lupus erythematosus; VCA, viral capsid antigen.

Increased levels of VCA IgG remained significantly associated with transitioning to SLE after additionally adjusting the models for ANA positivity (OR $1.2295 \%$ CI 1.01 to $1.47, \mathrm{p}=0.04$ ). EA IgG levels were also associated with transitioning to SLE (table 2) when compared with non-transitioned relatives. No significant interactions were found between the anti-EBV antibody levels and age, sex or race.

Increasing levels of VCA IgG and EA IgG were associated with transitioning to SLE when compared with controls (table 2), and the association remained after additional adjustment for ANA positivity (OR 1.46, 95\% CI 1.09 to $1.97, \mathrm{p}=0.01$ and OR 2.00 $95 \%$ CI 1.21 to $3.31, \mathrm{p}=0.007$, respectively). Furthermore, EA IgG positivity was associated with transitioning to SLE (OR 4.35 $95 \%$ CI 2.03 to $9.31, \mathrm{p}=0.0002$ ) when compared with controls. Levels of CMV IgG and HSV-1 IgG were not associated with transitioning to SLE (table 2).

\section{Variants in IL10 and CD40 interact with anti-VCA antibodies in transitioning to SLE}

Recent studies show a close association between environmental and host genetic factors in the development or exacerbation of the autoimmune response. ${ }^{28} 29$ To test whether there is an association between immune response to EBV and variants in host genes implicated in EBV infection, we determined the interactions between variants in IL10, CD40 and CR2 with anti-EBV responses in transitioning to SLE. We also tested interactions between anti-EBV responses and variants in TNFAIP3, a gene associated with SLE but not implicated in EBV infection.

IL10 variants were associated with transitioning to SLE (table 3); however, no association was observed with the CD40 variants or CR2 variant in this small cohort.

The association between VCA IgG level and transitioning to SLE was modified by CD40 rs4810485 (interaction $\mathrm{p}=0.0009$ ) (figure 3A). Increased VCA IgG level was associated with increased SLE risk in relatives with 0 minor alleles $(\mathrm{OR}=1.62$, 95\% CI 1.22 to 2.14), adjusting for age, sex and race (figure $3 \mathrm{~A}$ ). Similarly, the association between VCA IgA and transitioning to SLE was modified by IL10 rs3024493 (interaction $\mathrm{p}=0.008$ ) (figure 3C). Although neither VCA IgA levels nor the SNP in CR2 were associated with transitioning to SLE independently, we observed a modest but significant interaction between VCA IgA response and the CR2 variant rs17615 in SLE transition risk (interaction $\mathrm{p}=0.03$ ) (see online supplementary table 2 ). Similar results were found when comparing transitioned relatives to controls (figure 3B,D). We also tested association of SNPs in TNFAIP3 as a gene associated with SLE but not related to EBV. SNPs rs5029939 (in LD with rs5029937, rs2230926) and rs5029930 (in LD with rs3757173) were not associated with transitioning to SLE (table 3). Furthermore, there were no significant interactions between TNFAIP3 SNPs and viral antibodies in transitioning to SLE.

\section{DISCUSSION}

In this study, we evaluated the contribution of EBV reactivation to transitioning to SLE in unaffected relatives of patients with SLE. Our data show that SLE relatives had increased reactivation of EBV prior to transitioning to SLE and increasing levels of EBV antibodies associated with SLE disease transitioning.

EBV infection has been linked to autoantibody production through molecular mimicry and epitope spreading. Aberrant antibody responses against Epstein-Barr Nuclear Antigen-1 (EBNA-1) can incite antibody responses against lupus autoantigens, Sm and Ro. ${ }^{30}$ Anti-Ro responses were shown to be

Table 3 SNPs in IL10 are associated with transitioning to SLE

\begin{tabular}{|c|c|c|c|c|c|}
\hline Gene & SNP & Minor allele & MAF & $\begin{array}{l}(n=354) \\
\text { OR }(95 \% \text { CI) p value }\end{array}$ & $\begin{array}{l}\text { unaffected controls }(n=115) \\
\text { OR }(95 \% \mathrm{Cl}) \text { p value }\end{array}$ \\
\hline IL10 & rs3024505 & $\mathrm{T}$ & 0.14 & $2.15(1.31$ to 3.53$) 0.002$ & $1.87(1.03$ to 3.39$) 0.04$ \\
\hline IL10 & rs3024495 & A & 0.15 & 2.13 (1.31 to 3.45 ) 0.002 & 2.00 (1.09 to 3.65$) 0.02$ \\
\hline $1 L 10$ & rs3024493 & $\mathrm{T}$ & 0.14 & 2.06 (1.25 to 3.37$) 0.004$ & $1.91(1.05$ to 3.50$) 0.03$ \\
\hline IL10 & rs1800896 & $\mathrm{C}$ & 0.45 & $1.15(0.77$ to 1.72$) 0.50$ & $1.23(0.74$ to 2.06$) 0.43$ \\
\hline IL10 & rs3122605 & G & 0.13 & 1.86 (1.08 to 3.21$) 0.02$ & 1.79 (0.98 to 3.27$) 0.06$ \\
\hline$C D 40$ & rs1569723 & G & 0.43 & $0.98(0.72$ to 1.33$) 0.91$ & $0.86(0.59$ to 1.23$) 0.40$ \\
\hline CD40 & rs1883832 & A & 0.24 & 1.02 (0.62 to 1.68$) 0.93$ & 1.11 (0.63 to 1.94$) 0.73$ \\
\hline CD40 & rs4810485 & A & 0.25 & 0.95 (0.57 to 1.59$) 0.85$ & $1.03(0.59$ to 1.80$) 0.90$ \\
\hline$C R 2$ & rs17615 & $\mathrm{T}$ & 0.31 & 0.97 (0.61 to 1.53 ) 0.88 & 0.72 (0.42 to 1.24$) 0.24$ \\
\hline TNFAIP3 & rs5029930 & $\mathrm{C}$ & 0.14 & 1.41 (0.78 to 2.55$) 0.25$ & $1.49(0.79$ to 2.81$) 0.22$ \\
\hline TNFAIP3 & rs5029939 & G & 0.05 & $1.19(0.572 .49) 0.65$ & $1.96(0.82$ to 4.68$) 0.13$ \\
\hline
\end{tabular}

Statistically significant values $(p<0.05)$ are in bold. 

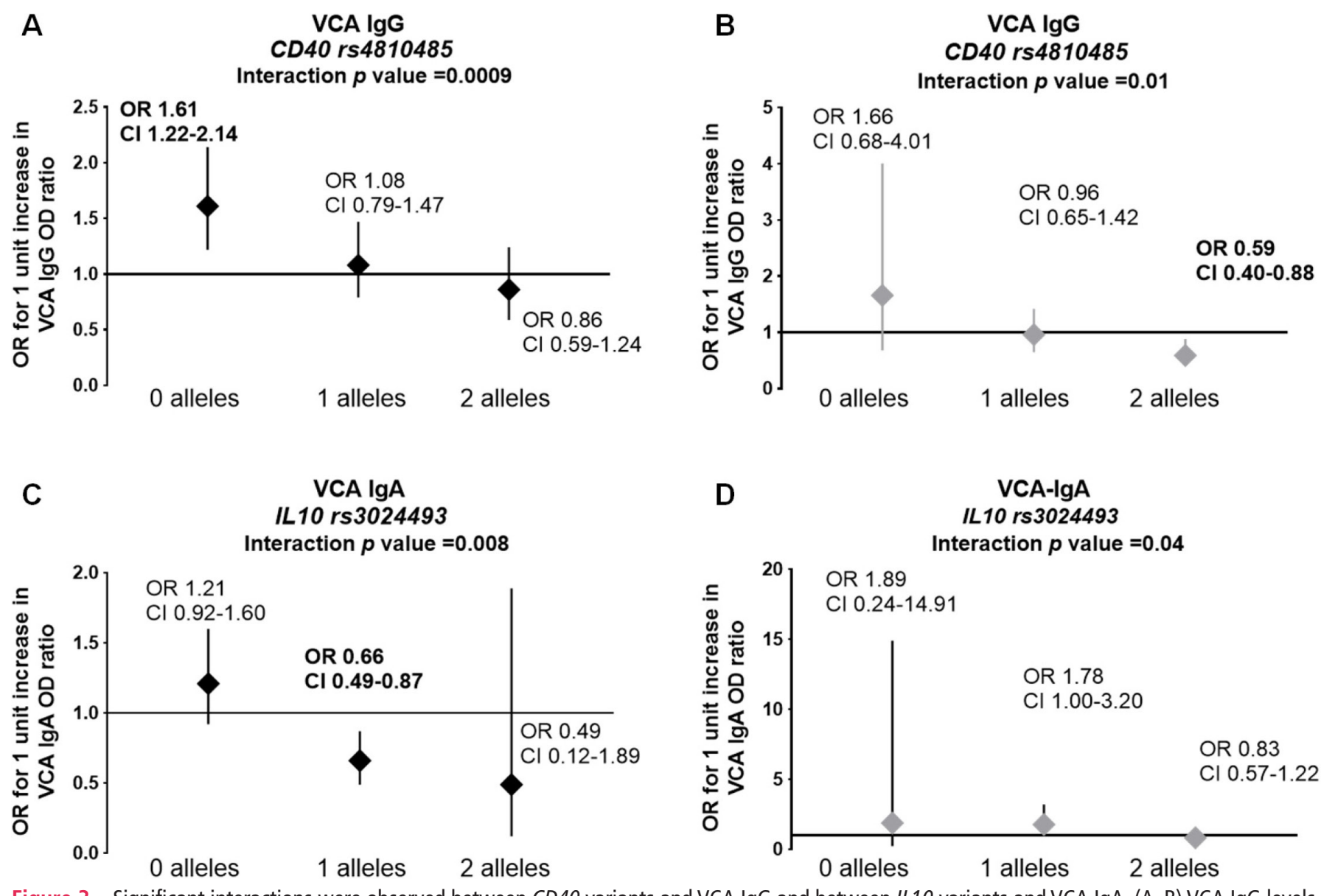

Figure 3 Significant interactions were observed between CD40 variants and VCA IgG and between IL10 variants and VCA IgA. (A, B) VCA IgG levels show significant interactions with CD40 variant rs 4810485 in the risk of transitioning to SLE. CD40 rs 4810485 : Transition $n=51$, relatives who did not transition $n=355$, unaffected unrelated controls $n=115$. (A) Interaction $p$ value: $p=0.0009$ when compared with relatives who did not transition. (B) Interaction $p$ value $p=0.01$ when compared with controls. (C,D) Significant interactions were observed between VCA IgA and IL10 variant rs 3024493 in the risk of transitioning to SLE. IL10 rs3024493: Transition $n=52$, relatives who did not transition $n=354$, unrelated unaffected controls $n=116$. (C) Interaction $p$ value $=0.008$ when compared with those who did not transition. (D) Interaction $p$ value $p=0.04$ when compared with controls. SLE, systemic lupus erythematosus; VCA, viral capsid antigen.

among the first autoantibodies detected preceding SLE classification in longitudinal samples. ${ }^{32}$ In this study, we show that the transitioned relatives had increased VCA IgG and EA IgG responses compared with non-transitioned relatives who were ANA- at baseline. There were significant differences between VCA IgG and EA IgG levels in the non-transitioned relatives based on their ANA positivity, and ANA+ relatives had significantly higher EBV antibody levels compared with ANA- relatives. In addition, differences in VCA IgA between transitioned and non-transitioned groups became significant when accounting for IL10 rs3024493. These data support that EBV reactivation may contribute to autoimmune responses through facilitating the loss of tolerance to lupus autoantigens. A prospective natural history study of high-risk individuals with serial evaluations and sample collection is needed to precisely define the timing of SLE transition relative to EBV reactivation and to perform a trajectory analysis over time.

We observed higher seroprevalence and higher levels of CMV IgG and HSV-1 IgG in unaffected controls compared with lupus relatives. CMV seroprevalance shows racial/ethnic differences ${ }^{33}$ and is influenced by socioeconomic status. ${ }^{34}$ The controls used in this study were recruited from community health centres where the exposure levels may be impacted by various factors including socioeconomic status. Levels of IgG directed against CMV and
HSV-1 antigens did not show any significant differences between transitioned and non-transitioned relatives. Furthermore, CMV IgG and HSV-1 IgG were not associated with transitioning to SLE. We also did not observe any differences in IgG responses towards varicella zoster (see online Supplementary Figure S2). The immune response to EBV is therefore unique in those who transitioned and is related to ANA positivity.

We observed increased levels of both VCA IgG and EA IgG in transitioned, as well as non-transitioned, SLE relatives when compared with controls. When transitioned relatives were compared with either non-transitioned relatives or controls, the association between VCA IgG levels and transitioning to SLE was modified by SLE-associated SNPs in CD40 rs4810485, and VCA IgA levels were associated with increased risk of transitioning in individuals with 0 minor alleles at IL10 rs3024493. These data suggest that the genetic predisposition to SLE, as is expected in relatives of patients with SLE, influences the immune response to a latent EBV infection, and that the increased reactivation of EBV in the context of host genetic risk alleles may increase the risk of transitioning to SLE. However, the exact relationship between EBV antibodies and SLE risk alleles remains unclear. The presence of risk alleles may allow for a frequent reactivation of EBV through as yet unknown mechanisms. 
Our data show that increased serological reactivation of EBV prior to SLE classification is associated with transitioning to SLE in genetically susceptible individuals. The negative predictive value for EA IgG positivity in SLE relatives was 89.33\% and is possibly better than the currently available measures for assessing risk of transitioning in SLE relatives. We also show that there are significant interactions between SLE-associated variants in host genes implicated in viral-related pathways and measures of EBV serological reactivation. To our knowledge this is the first prospective study examining preclinical association between serological measures of EBV reactivation and SLE disease transition.

\author{
Author affiliations \\ 'Arthritis and Clinical Immunology Program, Oklahoma Medical Research \\ Foundation, Oklahoma City, Oklahoma, USA \\ 2Department of Epidemiology, University of Colorado Anschutz Medical Campus, \\ Colorado School of Public Health, Aurora, Colorado, USA \\ ${ }^{3}$ Department of Medicine, Medical University of South Carolina, Charleston, South \\ Carolina, USA \\ ${ }^{4}$ Division of Rheumatology, Cedars-Sinai Medical Center, Los Angeles, California, USA \\ ${ }^{5}$ Division of Rheumatic Diseases, University of Texas Southwestern Medical Center, \\ Dallas, Texas, USA \\ ${ }^{6}$ Center for Autoimmune Genomics and Etiology, Cincinnati Children's Hospital \\ Medical Center, Cincinnati, Ohio, USA \\ ${ }^{7}$ Department of Pediatrics, University of Cincinnati College of Medicine, Cincinnati, \\ $\mathrm{OH}$, United States \\ ${ }^{8}$ US Department of Veterans Affairs Medical Center, Cincinnati, $\mathrm{OH}$, United States \\ ${ }^{9}$ Departments of Medicine and Pathology, University of Oklahoma Health Sciences \\ Center, Oklahoma City, Oklahoma, USA
}

Acknowledgements The authors would like to thank the Lupus Family Registry and Repository (Oklahoma Medical Research Foundation (OMRF)) and Systemic Lupus Erythematosus in Gullah Health (Medical University of South Carolina) groups, as well as Virginia Roberts and Tiny Powe for their assistance in recruiting study participants. The authors would also like to thank the Oklahoma Clinical Characterization and Biorepository Core and the OMRF Clinical Genomics Center for technical assistance and Kandice L Tessneer, PhD for editorial assistance.

Contributors All authors contributed to drafting or critically revising the article for intellectual content and approved the final version of the article. JAJ had access to all data and analyses.Study conception and design: NRJ, KAY, MEM, JMG, DLK, MW, DJW, GG, DRK, JBH, JMN and JJ. Acquisition of data. NRJ, MTH, JMG, DLK, MHW, $D W, G G, D R K, P M G, J B H$ and JAJ. Analysis and interpretation of data. NRJ, KAY, MEM, MTH, JMG, DLK, MHW, DW, GG, DRK, JAK, JBH, JMN and JJ.

Funding This study was supported by the National Institute of General Medical Sciences (U54GM104938 [JAJ]), the National Institute of Arthritis, Musculoskeletal and Skin Diseases (P30AR053482 [JAJ], P30AR072582 [GG], P60AR062755 [GG and DLK], K24AR068406 [DLK], P50AR055503 [DRK]), the National Institute of Allergy and Infectious Diseases (U19AI082714 [JAJ], U01Al101934 [JAJ], R01AI024717 [JBH], U01Al130830 [JBH]), the National Human Genome Research Institute (U01HG008666 [JBH]) and the Department of Veterans Affairs (JBH). The content is solely the responsibility of the authors and does not necessarily represent the official views of the National Institutes of Health or the US Government.

Competing interests Authors report funding from the National Institutes of Health. JBH has pending patents on broadly related work.

Patient consent for publication Not required.

Provenance and peer review Not commissioned; externally peer reviewed.

Data sharing statement All data relevant to the study are included in the article or uploaded as supplementary information.

\section{REFERENCES}

1 Chen L, Morris DL, Vyse TJ. Genetic advances in systemic lupus erythematosus: an update. Curr Opin Rheumatol 2017;29:423-33.

2 Deng Y, Tsao BP. Updates in lupus genetics. Current Rheumatology Reports 2017;19.

3 Block SR, Winfield JB, Lockshin MD, et al. Studies of twins with systemic lupus erythematosus. A review of the literature and presentation of 12 additional sets. Am J Med 1975;59:533-52.

4 Deapen $D$, Escalante A, Weinrib L, et al. A revised estimate of twin concordance in systemic lupus erythematosus. Arthritis Rheum 1992;35:311-8.

5 Gulley ML. Molecular diagnosis of Epstein-Barr virus-related diseases. J Mol Diagn 2001;3:1-10.
6 James JA, Kaufman KM, Farris AD, et al. An increased prevalence of Epstein-Barr virus infection in young patients suggests a possible etiology for systemic lupus erythematosus. J Clin Invest 1997;100:3019-26.

7 James JA, Neas BR, Moser KL, et al. Systemic lupus erythematosus in adults is associated with previous Epstein-Barr virus exposure. Arthritis Rheum 2001:44:1122-6.

8 Hanlon P, Avenell A, Aucott L, et al. Systematic review and meta-analysis of the sero-epidemiological association between Epstein-Barr virus and systemic lupus erythematosus. Arthritis Res Ther 2014;16.

9 Gross AJ, Hochberg D, Rand WM, et al. EBV and systemic lupus erythematosus: a new perspective. J Immunol 2005;174:6599-607.

10 Kang I, Quan T, Nolasco H, et al. Defective control of latent Epstein-Barr virus infection in systemic lupus erythematosus. J Immunol 2004;172:1287-94.

11 Huggins ML, Todd I, Powell RJ. Reactivation of Epstein-Barr virus in patients with systemic lupus erythematosus. Rheumatol Int 2005;25:183-7.

12 Esen BA, Y.lmaz G, Uzun S, et al. Serologic response to Epstein-Barr virus antigens in patients with systemic lupus erythematosus: a controlled study. Rheumatol Int 2012;32:79-83.

13 Fingeroth JD, Weis JJ, Tedder TF, et al. Epstein-Barr virus receptor of human B lymphocytes is the C3d receptor CR2. Proceedings of the National Academy of Sciences 1984;81:4510-4.

14 Tanner J, Weis J, Fearon D, et al. Epstein-Barr virus gp350/220 binding to the B lymphocyte C3d receptor mediates adsorption, capping, and endocytosis. Cell 1987;50:203-13.

15 Hsu D, de Waal Malefyt R, Fiorentino D, et al. Expression of interleukin-10 activity by Epstein-Barr virus protein BCRF1. Science 1990;250:830-2.10.1126/ science. 2173142

16 Jog NR, Chakravarty EF, Guthridge JM, et al. Epstein Barr virus interleukin 10 suppresses anti-inflammatory phenotype in human monocytes. Front Immunol 2018;9.

17 Uchida Jet al. Mimicry of CD40 signals by Epstein-Barr virus LMP1 in B lymphocyte responses. Science 1999;286:300-3.

18 Imadome K-I, Shirakata M, Shimizu N, et al. CD40 ligand is a critical effector of Epstein-Barr virus in host cell survival and transformation. Proceedings of the National Academy of Sciences 2003;100:7836-40.

19 Sakurai D, Zhao J, Deng Y, et al. Preferential binding to Elk-1 by SLE-associated IL10 risk allele upregulates IL10 expression. PLoS Genetics 2013;9:e1003870.

20 Vazgiourakis VM, Zervou MI, Choulaki C, et al. A common SNP in the CD40 region is associated with systemic lupus erythematosus and correlates with altered CD40 expression: implications for the pathogenesis. Ann Rheum Dis 2011;70:2184-90.

21 Chen J-M, Guo J, Wei C-D, et al. The association of CD40 polymorphisms with CD40 serum levels and risk of systemic lupus erythematosus. BMC Genetics 2015;16.

22 Douglas KB, Windels DC, Zhao J, et al. Complement receptor 2 polymorphisms associated with systemic lupus erythematosus modulate alternative splicing. Genes Immun 2009;10:457-69.

$23 \mathrm{Wu} \mathrm{H}$, Boackle SA, Hanvivadhanakul P, et al. Association of a common complement receptor 2 haplotype with increased risk of systemic lupus erythematosus. Proceedings of the National Academy of Sciences 2007:104:3961-6.

24 Rasmussen A, Sevier S, Kelly JA, et al. The lupus family registry and Repository. Rheumatology 2011:50:47-59.

25 Kamen DL, Barron M, Parker TM, et al. Autoantibody prevalence and lupus characteristics in a unique African American population. Arthritis Rheum 2008;58:1237-47.

26 Bruner BF, Guthridge JM, Lu R, et al. Comparison of autoantibody specificities between traditional and bead-based assays in a large, diverse collection of patients with systemic lupus erythematosus and family members. Arthritis Rheum 2012;64:3677-86

27 Munroe ME, Young KA, Kamen DL, et al. Discerning risk of disease transition in relatives of systemic lupus erythematosus patients utilizing soluble mediators and clinical features. Arthritis Rheumatol 2017;69:630-42.

28 Young KA, Munroe ME, Guthridge JM, et al. Combined role of vitamin D status and CYP24A1 in the transition to systemic lupus erythematosus. Ann Rheum Dis 2017:76:153-8.

29 Takvorian SU, Merola JF, Costenbader KH. Cigarette smoking, alcohol consumption and risk of systemic lupus erythematosus. Lupus 2014;23:537-44.

30 Kaufman KM, Kirby MY, Harley JB, et al. Peptide mimics of a major lupus epitope of SmB/B'. Ann N Y Acad Sci 2003;987:215-29.

31 McClain MT, Heinlen LD, Dennis GJ, et al. Early events in lupus humoral autoimmunity suggest initiation through molecular mimicry. Nature Medicine 2005;11:85-9.

32 Arbuckle MR, McClain MT, Rubertone MV, et al. Development of autoantibodies before the clinical onset of systemic lupus erythematosus. N Eng/ J Med 2003:349:1526-33.

33 Staras SAS, Dollard SC, Radford KW, et al. Seroprevalence of cytomegalovirus infection in the United States, 1988-1994. Clin Infect Dis 2006:43:1143-51.

34 Dowd JB, Aiello AE, Alley DE. Socioeconomic disparities in the seroprevalence of cytomegalovirus infection in the US population: NHANES III. Epidemiol Infect 2009;137:58-65. 